\title{
SREATE
}

Centre de Recherche en économie de l'Environnement, de l'Agroalimentaire, des Transports et de l'Énergie
Center for Research on the economics of the Environment, Agri-food, Transports and Energy

\section{The Effects of the Length of the Period of Commitment on the Size of Stable International Environmental}

\author{
Agreements
}

Bruno Nkuiya

Cahier de recherche/Working Paper 2012-7

Août/August 2012

Nkuiya: Department of Economics and CREATE, Université Laval

Please address correspondence to: Département d'économique, pavillon J.-A.-DeSève, Office 2244, 1025, avenue des SciencesHumaines, Québec, QC Canada G1V 0A6

robeny-bruno.nkuiya-mbakop.1@ulaval.ca

I am grateful to Gérard Gaudet, Markus Herrmann, Santiago Rubio, Scott Barrett for their comments on earlier drafts of this paper.

Les cahiers de recherche du CREATE ne font pas l'objet d'un processus d'évaluation par les pairs/CREATE working papers do not undergo a peer review process.

ISSN 1927-5544 


\begin{abstract}
This paper extends the standard model of self-enforcing dynamic international environmental agreements by allowing the length of the period of commitment of such agreements to vary as a parameter. It analyzes the pattern of behavior of the size of stable coalitions, the stock of pollution, and the emission rate as a function of the length of the period of commitment. It is shown that the length of the period of commitment can have very significant effects on the equilibrium. We show numerically that at the initial date, as the length of commitment is increased, the potential gain from cooperation tends to diminish, increasing the disincentive to ratify the agreements. This suggests that considerable attention should be given to the determination of the length of such international agreements.
\end{abstract}

JEL classification: Q5; C73; F53

Keywords: International Environmental Agreements; Global pollution; Stock pollution; Dynamic games

\title{
Résumé
}

Ce papier étend la classe des modèles dynamiques standards traitant des accords internationaux sur l'environnement au cas où la durée de la période d'engagement à de tels accords est un paramètre variable. Nous y étudions les évolutions dans le temps de la taille des coalitions stables, du stock de pollution et du taux d'émissions en fonction de la durée d'engagement. Nous prouvons que la longueur de la période d'engagement a un effet très significatif sur l'équilibre. À la période initiale, lorsque la période d'engagement est augmentée, nous montrons numériquement que le gain potentiel de la coopération tend à diminuer réduisant l'incitation à ratifier les accords. Ces faits portent à croire qu'il faudrait accorder une attention toute particulière au choix de la durée d'engagement lors de l'élaboration de tels accords internationaux.

Classification JEL: Q5; C73; F53

Mots-clés: Accords internationaux sur l'environnement; Pollution globale; Pollution par les stocks; Jeux dynamiques 


\section{Introduction}

In many contexts, International Environmental Agreements (IEAs) necessarily involve dynamic considerations. This is because they have to deal with stock pollutants and involve interactions over time among countries. Two approaches have been adopted in modeling such agreements. One consists in assuming that membership and emission strategies of the signatories and non-signatories are determined once and for all at the outset, with each of the signatories and non-signatories committing to an infinite path of emissions. Another consists in analyzing the problem in a discrete-time framework and assuming that membership and emission decisions are revised every period.

These two formulations correspond to two very particular assumptions about the length of the period of time for which the countries are required to commit. In reality the length of the period of commitment can be an important element of negotiation, and the resulting equilibrium may well depend significantly on this length. Intuitively, one might think that a short period of commitment could favor a larger coalition size than a longer one, since the parties will then have the option of revising their membership and emission decisions more frequently, after having observed the state that results at the close of the previous agreement. The purpose of this paper is to analyze the effect of varying the length of the period of commitment on the size and stability of such IEAs.1

The model used is closely related to that of Rubio and Casino (2005) and Rubio and Ulph (2007). Rubio and Casino (2005) adapt to a dynamic framework the concept of IEA introduced by Barrett (1994) and Carraro and Siniscalco (1993). They assume that at the initial date, given the initial stock of pollution, countries play a two-stage game. In the first stage (the membership game), anticipating the play of the game in the second stage, the countries decide non-cooperatively whether or not to join the agreement. In the second stage (the

1 Reinganum and Stokey (1985) explore the impact of the length of the period of commitment on the optimal extraction of a common pool nonrenewable resource. They find that shrinking the length of the period of commitment leads to a quick depletion of the resource. However, they do not address the issue of coalition formation in their paper. 
emission game), each non-signatory decides non-cooperatively the emission rate that maximizes its discounted net benefit, taking as given the emission path of the other countries. Signatory countries jointly choose their emission paths, acting non-cooperatively against non-signatories in order to maximize their aggregate discounted net benefits. Signatories also take as given the strategy of non signatories. The coalition formed in the membership game cannot change in the emission game. Hence countries commit to both their membership or non membership decision and to their respective emission paths for a period of infinite length. Using numerical simulations, they find that a two-country coalition is the only self-enforcing IEA.

Rubio and Ulph (2007) extend that paper to an infinite-horizon model in a discrete-time framework. At the outset of each period, given the stock of pollution at the beginning of the period, the play of the game is as in the game described above. Countries commit to membership or non membership and to their respective emission strategies for the duration of the period, whose length is normalized to one as it is usually the case in discrete-time modeling. The authors find that, in this context, there exists a steady-state stock of pollution and a corresponding steady-state IEA membership size and that, in the transition towards this steady state, the membership size and the stock of pollution vary inversely.

In this paper, we adopt an infinite horizon continuous-time framework, but treat the length of the period of commitment as a parameter that can take any strictly positive value. It is thus possible to study the effect of exogenously varying the length of the period of commitment on the equilibrium size of the stable coalition and stock of pollution, as well as on their pattern of behavior over time. Except for the extreme case of a single period of commitment of infinite length, there will be an infinite number of periods of commitment, the length of which is exogenously given at the outset. At the beginning of every period of commitment, each country decides whether or not to adhere to the agreement. The signatories then jointly decide on their emission rate for the period of commitment, while the non-signatories make that decision unilaterally. 
It is first shown analytically that non-signatories always pollute more than signatories and that they always gain more than signatories from any agreement, irrespective of the length of the period of commitment. Numerical simulations are then used to show that the length of the period of commitment can have a very significant effect on the equilibrium. At the initial date, two critical values of the length of commitment appear to be relevant. A first critical value is shown to exist below which the model generates the highest level of cooperation. Above this critical value and below the next one, there is a negative relationship between membership size and the length of commitment. Above this second critical value, the equilibrium yields the smallest level of cooperation for the earlier period. These results may be attributed to the fact that reducing the length of commitment tends to increase the potential gain from cooperation.

The limiting case of a single period of commitment of infinite length is shown to yield the smallest possible coalition for the earlier period. This generates a lower gain from cooperation and a higher trajectory of the stock of pollution than in the case of some finite lengths of commitment.

The remainder of the paper is organized as follows. Section 2 sets out the model. Section 3 resolves the second stage of the game. In addition, the outcomes of the cooperative and the non-cooperative equilibria are derived in that section. Section 4 presents the first stage of the game. In Section 5, the importance of the choice of the length of the period of commitment is investigated by simulation. Section [6 concludes.

\section{The model}

Consider the formation of an infinite sequence of IEAs, in which countries can make binding commitments about their emission rates and their membership decision over a limited horizon. Define a period to be the interval of (continuous) time over which countries can make such commitments, and let $h$ be the length of the period. Assume an infinite number of such periods, $[0, h],[h, 2 h],[2 h, 3 h], \ldots$, and $N$ identical countries, $i=1, \ldots, N$. Each country 
makes a membership decision and commits to a level of emission for each of the intervals $[0, h],[h, 2 h],[2 h, 3 h], \ldots,$. We will assume that one unit of production generates one unit of emissions. Let $q_{i}$ denote the emissions of country $i$. Following Rubio and Ulph (2007), assume that at each instant $q_{i} \in[0,1]$. The current aggregate emissions of the world is then $Q=\sum_{i=1}^{N} q_{i} \in[0, N]$.

The current stock of pollution is denoted $z(t)$. We assume that the amount of pollutants emitted today by the world adds to the current stock of pollution according to the kinematic equation

$$
\dot{z}(t)=Q(t)-\rho z(t), \quad \rho \in(0,1) \quad z(0)=z_{0},
$$

where $\rho$ is the natural purification rate.

The stock of pollution at each date generates damage costs for each country that we assume to be a quadratic function of the stock: $\frac{\gamma}{2} z^{2}$, where $\gamma$ is a positive constant. As in Ulph (2004) and Rubio and Ulph (2007), the instantaneous benefit function is assumed to be linear in current emissions : $a q$, where $a$ is a positive constant. Thus, a country's flow of net benefits is given by

$$
\pi(q, z)=a q-\frac{\gamma}{2} z^{2}
$$

At the beginning of every period, each country determines an emission strategy for that period. A country's choice will depend on the beginning-of-period stock and the length of the period, $h$. Let $q_{j}^{k}\left(z_{k}\right)$ denote the emission strategy planned by country $j$ for period $k$ when the stock of pollution at the outset of the period is $z(k h)=z_{k}$.

The model of IEA formation in each period is a dynamic version of the model of selfenforcing IEAs introduced by Carraro and Siniscalco (1993) and Barrett (1994) and the continuous-time version of Rubio and Ulph (2007). At the beginning of each period, given the initial stock, there is a two-stage game. In the first stage (the membership game), countries first decide whether or not to join an IEA. In the second stage (the emission game), non-signatory countries choose their emissions for the current period non-cooperatively, while signatory countries act in a cooperative fashion. 
For example, for the period $[k h,(k+1) h]$, given the initial pollution stock of the current period $z_{k}$, countries play the two-stage game at the initial date $t=k h$ of the current period. The membership decision that results from the membership game and the emission strategy $q_{j}^{k}$ of a given country $j$ are thus decided at the initial date of the current period. For simplicity's sake, we will assume that it commits to a constant $q_{j}^{k}$ for the duration of period $k$ and we will restrict our attention to symmetric equilibria.

Let us first define how current membership decisions affect future ones. Following Rubio and Ulph (2007), Ulph (2004) and Nkuiva et al. (2011), we use the random assignment rule, which states that countries cannot commit to their membership across periods. We assume that there is a binomial random variable whose realization at any given period determines whether or not a particular country will be among the members for the period. For any stable IEA of size $n \leq N$ in that period, the a priori probability of any given country being a member of the coalition is $n / N$. Since we consider symmetric equilibria, this probability is the same for all countries and is independent of the history of membership decisions. Therefore each country has the same expected present value of current and future net benefits, which will depend on the initial pollution stock of the next period. We will denote by $\Psi\left(z_{k}\right)$ the expected present value of current and future net benefits of the representative country when the stock of pollution at the outset of the period is $z_{k}$.

In each period, the second stage of the game is solved first, taking as given the set of signatories of the membership game.

\section{The second stage of the game}

Consider some beginning of period date $t \in\{0, h, 2 h, 3 h, 4 h, \ldots\}$, when the stock of pollution is $z(t)$. Let $K(S)$ denote the set of signatories and $n$ the number of signatories at that date. The current value function of a non-signatory is then

$$
V_{j}(n, z(t))=\max _{q_{j} \in[0,1]}\left\{\int_{t}^{t+h} e^{-r(s-t)} \pi\left(q_{j}, z(s)\right) d s+e^{-r h} \Psi(z(t+h))\right\},
$$

subject to (11) and (21), where $r$ is the discount rate. 
The aggregate current value function of all signatories at the same date is

$$
V_{S}(n, z(t))=\max _{q_{i}, i \in K(S)}\left\{\int_{t}^{t+h} e^{-r(s-t)} \sum_{i \in K(S)} \pi\left(q_{i}, z(s)\right) d s+n e^{-r h} \Psi(z(t+h))\right\},
$$

again subject to (11)-(2) and $q_{i} \in[0,1]$, for all $i \in K(S)$.

Since we consider only symmetric equilibria, the value function of signatory $i$ is $V_{i}(n, z(t))=$ $V_{S}(n, z(t)) / n$, for all $i \in K(S)$.

Definition 1 In an infinite-duration game defined by (3) and (4), with the length of period $h$, an emission strategy for country $j$ is a sequence of functions $q_{j} \equiv\left\{q_{j}^{k}:[k h,(k+1) h] \times R_{+} \rightarrow\right.$ $\left.R_{+}\right\}_{k=0}^{\infty}$, where $q_{j}^{k}$ is a constant function of $s \in[k h, k h+h]$, for $k=0,1,2, \ldots$

This means that at the outset of periods, given the coalition formed in the membership game, each country chooses and commits to use a constant emission rate in the emission game.

This continuous-time problem can be transformed into a discrete-time one. Indeed, on a given interval $[k h,(k+1) h]$, the emission strategies of the players are constant and so is the aggregate emission of the world, $Q$. Hence, the solution of the differential equation (1), given the initial stock of pollution $z(k h)=z_{k}$, is:

$$
z(t)=\frac{Q}{\rho}+\left(z_{k}-\frac{Q}{\rho}\right) e^{-\rho(t-k h)} \quad \forall t \in[k h,(k+1) h] .
$$

So, at time $t=(k+1) h$, the dynamic evolution of the pollution stock between the outset of periods $k$ and $k+1$ is given by:

$$
z((k+1) h) \equiv z_{k+1}=f(\rho, h) Q+z_{k} e^{-\rho h},
$$

where $f(x, h)=\left(1-e^{-h x}\right) / x, \quad \forall x>0$. We adopt this notation in the remainder of the paper. As shown in Appendix $\mathrm{A}$, the following integral yields the net benefit function at each period, which depends on the length of the period:

$$
\int_{k h}^{(k+1) h} e^{-r(s-k h)} \pi(q, z(s)) d s=a q f(r, h)+D\left(Q, z_{k}\right),
$$


where $D\left(Q, z_{k}\right)=-\frac{\gamma}{2}\left[\left(\frac{Q}{\rho}\right)^{2} f(r, h)+\left(z_{k}-\frac{Q}{\rho}\right)^{2} f(r+2 \rho, h)+2 \frac{Q}{\rho}\left(z_{k}-\frac{Q}{\rho}\right) f(r+\rho, h)\right]$.

Substituting (6) and (7) into (3), we obtain the Bellman equation for non-signatories:

$$
V_{j}\left(n, z_{k}\right)=\max _{q_{j} \in[0,1]}\left\{a q_{j} f(r, h)+D\left(Q, z_{k}\right)+e^{-r h} \Psi\left(f(\rho, h) Q+z_{k} e^{-\rho h}\right)\right\} .
$$

Similarly, substitution of (66) and (17) into (4) yields the Bellman equation for all signatories:

$$
V_{S}\left(n, z_{k}\right)=\max _{q_{i}, i \in K(S)}\left\{\sum_{i \in K(S)} a q_{i} f(r, h)+n D\left(Q, z_{k}\right)+n e^{-r h} \Psi\left(Q f(\rho, h)+z_{k} e^{-\rho h}\right)\right\},
$$

subject to $q_{i} \in[0,1]$ for all $i \in K(S)$.

The best response of the countries results from the strategic behavior between signatories and non-signatories. As in Ulph (2004) and Rubio and Ulph (2007), we will restrict the set of parameters to be such that the dominant strategy for non-signatories at each period will be to pollute at the maximum level. The necessary and sufficient condition for this is:

$$
a f(r, h) \geq \lambda_{1} Q+z_{k} \lambda_{2}-f(\rho, h) e^{-r h} \Psi^{\prime}\left(Q f(\rho, h)+z_{k} e^{-\rho h}\right),
$$

where $\lambda_{1}=\frac{\gamma}{\rho^{2}}(f(r, h)+f(r+2 \rho, h)-2 f(r+\rho, h))$ and $\lambda_{2}=\frac{\gamma}{\rho}(f(r+\rho, h)-f(r+2 \rho, h))$.

The Kuhn-Tucker conditions for the optimization problem (9) are, for $i \in K(S) 2$

$$
\begin{aligned}
& {\left[a f(r, h)-n\left(\lambda_{1} Q+z_{k} \lambda_{2}-f(\rho, h) e^{-r h} \Psi^{\prime}\left(Q f(\rho, h)+z_{k} e^{-\rho h}\right)\right)-\mu_{i}\right] q_{i}=0 ; q_{i} \geq 0,} \\
& a f(r, h)-n\left(\lambda_{1} Q+z_{k} \lambda_{2}-f(\rho, h) e^{-r h} \Psi^{\prime}\left(Q f(\rho, h)+z_{k} e^{-\rho h}\right)\right)-\mu_{i} \leq 0, \\
& \left(1-q_{i}\right) \mu_{i}=0 ; \quad \mu_{i} \geq 0 ; \quad 1 \geq q_{i},
\end{aligned}
$$

where $\mu_{i}$ is the Kuhn-Tucker multiplier associated to the constraint $q_{i} \leq 1$. It follows that when $q_{i}\left(n, z_{k}\right)$ satisfies simultaneously (10)-(13) , a non-signatory's and a signatory's payoff will be respectively given by

$$
\begin{aligned}
& V_{j}\left(n, z_{k}\right)=a f(r, h)+D\left(Q\left(n, z_{k}\right), z_{k}\right)+e^{-r h} \Psi\left(f(\rho, h) Q\left(n, z_{k}\right)+z_{k} e^{-\rho h}\right), \\
& V_{i}\left(n, z_{k}\right)=a q_{i}\left(n, z_{k}\right) f(r, h)+D\left(Q\left(n, z_{k}\right), z_{k}\right)+e^{-r h} \Psi\left(Q\left(n, z_{k}\right) f(\rho, h)+z_{k} e^{-\rho h}\right),
\end{aligned}
$$

where, $Q \equiv Q\left(n, z_{k}\right)=n q_{i}\left(n, z_{k}\right)+(N-n)$.

\footnotetext{
${ }^{2}$ We could write these conditions with an additional multiplier for the constraint $q_{i} \geq 0$, however, we would get the same outcome.
} 
Proposition 1 The current emissions by signatories are always less than the current emissions by non-signatories, and the resulting payoff of non-signatories is always greater than that of signatories for $n \in[2, N-1]$.

Proof. By construction, we have $0 \leq q_{i}\left(n, z_{k}\right) \leq 1=q_{j}\left(n, z_{k}\right)$. Using (14) and (15), since $0 \leq q_{i}\left(n, z_{k}\right) \leq 1$, we get: $V_{j}\left(n, z_{k}\right)-V_{i}\left(n, z_{k}\right)=a f(r, h)\left(1-q_{i}\left(n, z_{k}\right)\right) \geq 0$.

Proposition 1 shows that non-signatories pollute more than signatories, and they also gain more than signatories from any agreement. These results are known in the literature. They have been shown, among others, by Rubio and Ulph (2007) in a discrete-time model, with the length of the period of commitment set equal to one, and by Rubio and Casino (2005), with a period of commitment of infinite length. This proposition proves that those results hold irrespective of the length of the period of commitment.

Before solving the first stage of the game, it is useful to study the particular cases of the non-cooperative equilibrium $(n=1)$ and the fully-cooperative equilibrium $(n=N)$.

\subsection{The non-cooperative equilibrium}

Assume that all $N$ countries decide non-cooperatively the emission strategy of the current period that maximizes their discounted net benefit, taking as given the current emission strategy of the other countries. The Bellman equation is then the special case of (8) for which $n=1$ and $\Psi=V_{j}, 3$ and we have the following result.

Proposition 2 Assume that

$$
z_{0} \leq \tilde{z} ; \quad a f(r, h) \geq N \lambda_{1}+\tilde{B} e^{-r h} f(\rho, h)+\tilde{z}\left[\lambda_{2}+\tilde{A} e^{-r h} f(\rho, h)\right] .
$$

In the non-cooperative equilibrium each country will emit $q_{j}=1$ for each period. The sequence of pollution stocks at the outset of each period, $\left\{z_{k}\right\}_{k=0}^{\infty}$, increases and converges asymptotically to a steady state

$$
\tilde{z}=\frac{N}{\rho} .
$$

\footnotetext{
$V_{i}$.

${ }^{3}$ Condition (18) shows that $\Psi=\left[n V_{i}+(N-n) V_{j}\right] / N$. Hence, for $n=1$, (10) - suggest that $\Psi=V_{j}=$
} 
The present discounted net welfare for any country $j$ is given by

$$
V_{j}\left(z_{0}\right)=-\tilde{A} z_{0}^{2} / 2-\tilde{B} z_{0}+\tilde{C}
$$

where $\tilde{A}=\gamma /(r+2 \rho)>0, \tilde{B}=N\left[\lambda_{2}+\tilde{A} f(\rho, h) e^{-h(r+\rho)}\right] /\left(1-e^{-h(r+\rho)}\right)>0$, and $\tilde{C}=$ $\left[a f(r, h)-N^{2} \lambda_{1} / 2-e^{-r h} N f(\rho, h)(\tilde{B}+\tilde{A} N f(\rho, h))\right] /\left(1-e^{-r h}\right)$.

Proof. For $n=1$, first we assume that $q_{j}\left(n, z_{k}\right)=1$, so that $Q\left(n, z_{k}\right)=N$. Using a quadratic guess, $\Psi=V_{j}=-\tilde{A} z^{2} / 2-\tilde{B} z+\tilde{C}$, the left-hand side and the right-hand side of (14) are then second degree polynomials in $z$. Equating their coefficient of powers of $z$, we get the values of $\tilde{A}, \tilde{B}$, and $\tilde{C}$. Since $z_{0} \leq \tilde{z}$, the sequence $\left\{z_{k}\right\}_{k=0}^{\infty}$ is increasing and it converges to $\tilde{z}$ which is its lowest upper bound. Now, for a sufficient condition to have $q_{j}\left(1, z_{k}\right)=1$ at each period, rewrite (10) for $\Psi=-\tilde{A} z^{2} / 2-\tilde{B} z+\tilde{C}$, to get

$$
a f(r, h) \geq Q \lambda_{1}+\tilde{B} e^{-r h} f(\rho, h)+z_{k+1} \tilde{A} e^{-r h} f(\rho, h)+z_{k} \lambda_{2} .
$$

In this inequality, substituting $Q, z_{k}$ and $z_{k+1}$ by their respective upper bounds $N, \tilde{z}$, and $\tilde{z}$, and rearranging, yields the second condition of (16). Thus if the inequalities in (16) hold, it will be optimal for each country to emit $q=1$ at every period.

\subsection{The cooperative equilibrium}

Suppose now that all the countries decide cooperatively the emission strategies of the current period that maximizes their aggregate discounted net benefit. The Bellman equation is then the particular case of (9) for which $n=N$ and $\Psi=V_{S} / N=V_{i} \cdot 4$ If the following condition holds:

$$
z_{0} \leq \bar{z} ; \quad a f(r, h) \leq N^{2}\left[\lambda_{1}+\bar{A} e^{-r h} f(\rho, h)^{2}\right]+N \bar{B} f(\rho, h) e^{-r h},
$$

it will be optimal for each country to pollute a quantity between zero and one. This implies the following result.

\footnotetext{
${ }^{4}$ Equation (18) indicates that $\Psi=\left[n V_{i}+(N-n) V_{j}\right] / N$. Hence, for $n=N$, (10)-(15) show that $\Psi=V_{i} \equiv V_{S} / N$.
} 
Proposition 3 Assume that the condition in (17) holds. In the fully-cooperative equilibrium, the sequence of pollution stocks at the outset of each period, $\left\{z_{k}\right\}_{k=0}^{\infty}$, converges to a steady state

$$
\bar{z}=\frac{a f(r, h)-\bar{B} N e^{-r h} f(\rho, h)}{N\left(\lambda_{2}+\bar{A} f(\rho, h) e^{-r h}\right)+\rho N\left[\lambda_{1}+\bar{A} f(\rho, h)^{2} e^{-r h}\right]}
$$

if and only if

$$
R_{N}=\frac{\lambda_{1} e^{-\rho h}-\lambda_{2} f(\rho, h)}{\lambda_{1}+\bar{A} f(\rho, h)^{2} e^{-r h}}>-1 .
$$

This convergence is monotone if and only if $R_{N}>0$. At any period, say $k$, the optimal emission strategy for a country is given by

$$
q_{i}\left(z_{k}\right)=\frac{a f(r, h)-N \bar{B} f(\rho, h) e^{-r h}-z_{k} N\left[\lambda_{2}+\bar{A} f(\rho, h) e^{-h(r+\rho)}\right]}{N^{2}\left[\lambda_{1}+\bar{A} f(\rho, h)^{2} e^{-r h}\right]} .
$$

The present discounted net welfare for a country is given by $V_{i}\left(z_{0}\right)=\left(-\bar{A} z_{0}^{2} / 2-\bar{B} z_{0}+\bar{C}\right) / N$, where $\bar{A}, \bar{B}$, and $\bar{C}$ are given in Appendix $B$.

Proof. See Appendix B.

Since at each period, the emission rate by a country from the non-cooperative equilibrium is always greater than that from the cooperative equilibrium, it is so for their associated pollution stocks. Thus, when the steady state of the pollution stock of the cooperative equilibrium exists, it is always lower than that of the non-cooperative equilibrium 5

\section{The first stage of the game}

To solve the membership game we use the notion of stability introduced by D'Aspremont et al. (1983) 6

\footnotetext{
${ }^{5}$ Notice that these results may also hold in the presence of uncertainty, risk, various types of preferences, and learning. For useful discussions of this argument, see among others Dockner and Long (1993), Ulph (2004), Nkuiva (2011), and Long (1992).

${ }^{6}$ We use the same concept of stability as Ulph (2004), and Rubio and Ulph (2007). Other concepts exist and have been used in the literature (see, for example, de Zeeuw (2008), and Pavlova (2010)).
} 
Definition 2 At the beginning of a period, say $k$, if the current stock of pollution is $z_{k}$, a coalition of signatories $K(S)$ of size $n^{*}$ is said to be stable, or self-enforcing, if and only if

$$
\begin{aligned}
& V_{i}\left(n^{*}, z_{k}\right) \geq V_{j}\left(n^{*}-1, z_{k}\right) \\
& V_{j}\left(n^{*}, z_{k}\right) \geq V_{i}\left(n^{*}+1, z_{k}\right) .
\end{aligned}
$$

The first inequality of Definition 2 is the internal stability condition. Its interpretation is that a signatory country cannot be better off by leaving the coalition, given that the other countries maintain their membership decision. The second inequality is the external stability condition. It means that a non-signatory cannot be better off by joining the coalition, given that the other countries maintain their membership decision.

Notice that the equilibrium coalition size $n^{*}$ depends on the length of the period of commitment $h$, the current stock of pollution $z_{k}$, and the remaining parameters of the model. To alleviate notation, it will be denoted from now on by $n^{*}\left(z_{k}\right)$ instead of $n^{*}\left(z_{k}, h\right)$ ]

Using the random assignment rule presented in the previous section, each country has $a$ priori the same probability $n^{*}\left(z_{k}\right) / N$ of being a signatory. Therefore the expected present value of future net benefits for each country is:

$$
\begin{aligned}
\Psi\left(z_{k}\right)= & \frac{n^{*}\left(z_{k}\right)}{N} V_{i}\left(n^{*}\left(z_{k}\right), z_{k}\right)+\left(1-\frac{n^{*}\left(z_{k}\right)}{N}\right) V_{j}\left(n^{*}\left(z_{k}\right), z_{k}\right) \\
= & a Q\left(n^{*}\left(z_{k}\right), z_{k}\right) f(r, h) / N+D\left(Q\left(n^{*}\left(z_{k}\right), z_{k}\right), z_{k}\right) \\
& +e^{-r h} \Psi\left(Q\left(n^{*}\left(z_{k}\right), z_{k}\right) f(\rho, h)+z_{k} e^{-\rho h}\right) .
\end{aligned}
$$

Recall that the value function $\Psi$ must satisfy simultaneously conditions (10)-(13) along with (18).

\subsection{Quadratic approximation of the value function $\Psi$}

Since $Q\left(n^{*}\left(z_{k}\right), z_{k}\right)$ is not linear in $z_{k}$, we can see from (18) that a quadratic function is not a plausible guess for $\Psi$. In Appendix $\mathrm{D}$, we present an algorithm for deriving a quadratic

\footnotetext{
${ }^{7}$ Notice that: $(a)$ many stable coalitions may exist and $(b)$ a coalition whose size is the greatest integer for which internal stability holds is also externally stable. We therefore consider the largest coalition size for which internal stability is satisfied to be self-enforcing.
} 
approximation, $-A z^{2} / 2-B z+C, A>0 ; B>0$, of the function $\Psi$. This algorithm is merely an adaptation to any $h>0$ of that used by Rubio and Ulph (2007) which works only for $h=1$.

Let us assume that

$$
\begin{aligned}
& a f(r, h) \geq N\left(\lambda_{1}+\lambda_{2} / \rho\right)+f(\rho, h) e^{-r h}(B+A N / \rho), \\
& N B f(\rho, h) e^{-r h}+N z_{0}\left[\lambda_{2}+A e^{-h(r+\rho)} f(\rho, h)\right] \geq a f(r, h) .
\end{aligned}
$$

In order to derive the equilibrium, we show in Appendix $\mathrm{C}$ the existence of three critical values of $n$. They are defined by the formulae:

$$
\begin{aligned}
& \bar{n}_{\ell}\left(z_{k}\right)=\frac{\tau\left(z_{k}, h\right)+(-1)^{\ell} \sqrt{\tau\left(z_{k}, h\right)^{2}-4 a f(r, h)\left(\lambda_{1}+A e^{-r h} f(\rho, h)^{2}\right)}}{2\left(\lambda_{1}+A e^{-r h} f(\rho, h)^{2}\right)}, \ell \in\{1,2\}, \\
& \bar{n}_{0}\left(z_{k}\right)=a f(r, h) / \tau\left(z_{k}, h\right),
\end{aligned}
$$

where $\tau\left(z_{k}, h\right)=N \lambda_{1}+f(\rho, h) e^{-r h}(B+A N f(\rho, h))+z_{k}\left(\lambda_{2}+A f(\rho, h) e^{-h(r+\rho)}\right)$ and $z_{k}$ being the stock of pollution at the outset of period $k$.

Notice that when conditions (19) and (20) hold, we prove in Appendix C that:

$1 \leq \bar{n}_{0}\left(z_{k}\right)<\bar{n}_{1}\left(z_{k}\right)<N<\bar{n}_{2}\left(z_{k}\right)$. The following proposition characterizes the decision rules of emissions by signatories as well as non-signatories for each period.

Proposition 4 Assume that conditions (19) and (20) hold. The dominant strategy for nonsignatories is to emit $q_{j}=1$ at each period, irrespective of the coalition size and the stock of pollution. At any period, say $k$, if $z_{k}$ is the current stock of pollution, the best emission strategy for a signatory in a coalition of size $n$ is given by

$$
q_{i}\left(n, z_{k}\right)= \begin{cases}1 & \text { if } 1 \leq n \leq \bar{n}_{0}\left(z_{k}\right) \\ \frac{a f(r, h)+n^{2}\left(\lambda_{1}+A e^{-r h} f(\rho, h)^{2}\right)-n \tau\left(z_{k}, h\right)}{n^{2}\left(\lambda_{1}+A e^{-r h} f(\rho, h)^{2}\right)} & \text { if } \bar{n}_{0}\left(z_{k}\right) \leq n \leq \bar{n}_{1}\left(z_{k}\right) \\ 0 & \text { if } \bar{n}_{1}\left(z_{k}\right) \leq n \leq N\end{cases}
$$

where $\bar{n}_{0}\left(z_{k}\right)$ is given by (22) while $\bar{n}_{1}\left(z_{k}\right)$ and $\bar{n}_{2}\left(z_{k}\right)$ are given by (21).

Proof. See Appendix C.

Proposition 4 shows that as the size of the coalition increases 8 the emission rate of members

\footnotetext{
${ }^{8}$ Proposition 4 represents a generalization of results in Rubio and Ulph (2007), where $h=1$ necessarily.
} 
from that coalition decreases. Making use of (23), we get an analogous result for the global emission which decreases as the coalition size increases.

\subsection{Dynamics of the stock and number of signatory}

In this section, we explain how we use the above approximation of the value function to derive numerically the evolution of the stock of pollution and the number of signatories over time. In order to derive the equilibrium, for a fixed value of $h$, we consider the values of $A$, $B, C$ given by the algorithm for which inequalities (19) and (20) hold. Given $z_{k}$, we compute $n^{*}\left(z_{k}\right)$ and $q_{i}\left(n^{*}\left(z_{k}\right), z_{k}\right)$ using the steps $(i v)$ and $(v)$ of the algorithm. We compute $z(t)$ for $t \in(k h,(k+1) h)$ making use of (5). Given $z_{k}$ and $n^{*}\left(z_{k}\right)$, we calculate the stock of pollution at the outset of period $k+1$ according to:

$$
z_{k+1}=f(\rho, h)\left(N-n^{*}\left(z_{k}\right)\right)+f(\rho, h) n^{*}\left(z_{k}\right) q_{i}\left(n^{*}\left(z_{k}\right), z_{k}\right)+z_{k} e^{-\rho h}
$$

If the steady state of the pollution stock exists, (24) shows that it must depend on steadystate emissions by signatories. If the steady state of emissions by signatories is equal to zero, then $z^{\text {stea }}=\left(N-n^{*}\left(z^{\text {stea }}\right)\right) / \rho$. If the steady-state emissions by signatories are given by

$$
q_{i}\left(n^{*}\left(z^{\text {stea }}\right), z^{\text {stea }}\right)=\frac{a f(r, h)+n^{*}\left(z^{\text {stea }}\right)^{2}\left(\lambda_{1}+A e^{-r h} f(\rho, h)^{2}\right)-n^{*}\left(z^{\text {stea }}\right) \tau\left(z^{\text {stea }}, h\right)}{n^{*}\left(z^{\text {stea }}\right)^{2}\left(\lambda_{1}+A e^{-r h} f(\rho, h)^{2}\right)}
$$

then, solving the equation $z^{\text {stea }}=f(\rho, h)\left(N-n^{*}\left(z^{\text {stea }}\right)\right)+f(\rho, h) n^{*}\left(z^{\text {stea }}\right) q_{i}\left(n^{*}\left(z^{\text {stea }}\right), z^{\text {stea }}\right)+$ $z^{\text {stea }} e^{-\rho h}$ with respect to $z^{\text {stea }}$ yields

$$
z^{\text {stea }}=\frac{a f(r, h)-n^{*}\left(z^{\text {stea }}\right) B e^{-r h} f(\rho, h)}{n^{*}\left(z^{\text {stea }}\right)\left[\rho\left(\lambda_{1}+A e^{-r h} f(\rho, h)^{2}\right)+\left(\lambda_{2}+A e^{-h(r+\rho)} f(\rho, h)\right)\right]} .
$$

Finally, if the steady state of the emission level by signatories is equal to one, then the steady state of the pollution stock is $N / \rho$. This case is analogous to the long-run equilibrium for the non-cooperative equilibrium characterized by Proposition 2.

Notice that by setting $n^{*}\left(z^{\text {stea }}\right)=N$ in formulae (25) $-(26)$, one gets the same results as in the cooperative equilibrium. 


\section{Numerical simulations: the effects of the length of commitment}

In this section, we present the outcome of the numerical analysis on a set of $N=20$ identical countries with $a=367$ and $z_{0}=900$. We use the same parameter values as Rubio and Casino (2005). These are $r=0.025 ; \gamma=0.001$ and $\rho=0.005$. The model captures certain results of Rubio and Ulph (2007) for some values of the length of commitment and yields different results for other values.

The simulations have been carried out for thousand values of the length of commitment.9 What first appears clearly is that the current value function of signatories as well as of non-signatories increases with the number of signatories. Of greater interest is the effect of the length of commitment on the equilibrium number of signatories and on the gains from cooperation, to which we now turn.

\subsection{The length of commitment and the size of self-enforcing coalitions}

As concerns the size of self-enforcing coalitions, our simulations reveal that for all values of the parameter considered in this study, the size of the stable coalition $n^{*}\left(z_{k}, h\right)$ is the least integer greater than or equal to $n_{0}\left(z_{k}, h\right)$. The rationale is that if a member of the coalition $n^{*}\left(z_{k}, h\right)$ unilaterally decides to leave that coalition, members of the remaining coalition size $n^{*}\left(z_{k}, h\right)-1$ must play the same maximum level of emissions as the defector and therefore must have the same payoff. That action is not profitable because the larger the coalition, the greater the associated gain to its members. Moreover, we have found that the interval $\left[n_{0}\left(z_{k}, h\right), n_{1}\left(z_{k}, h\right)\right]$ contains at most one integer. These results suggest that members of a self-enforcing IEAs never emit the maximum emission as do non-members.

Our numerical analysis also highlights two critical values of the length of commitment $(\underline{h}=182.6$ and $\bar{h}=194.8)$, which distinguish two possible cases for the dynamics of the relation between membership size and the pollution stock. The first case corresponds to lengths of the period of commitment $(h<182.6$ or $h>194.8)$. In this case, as in Rubio and Ulph

\footnotetext{
${ }^{9}$ These value are obtained from the arithmetic sequence $h_{p}=h_{p-1}+0.1 ; h_{0}=0.1, p=1, \ldots, 2499$.
} 
(2007), our simulations suggest that the stock of pollution rises and converges asymptotically to its steady state, while the coalition size decreases over time and reaches its steady state after a finite number of periods. This is illustrated in Figure 1 for the case where the length of the period of commitment is equal to $h=110$
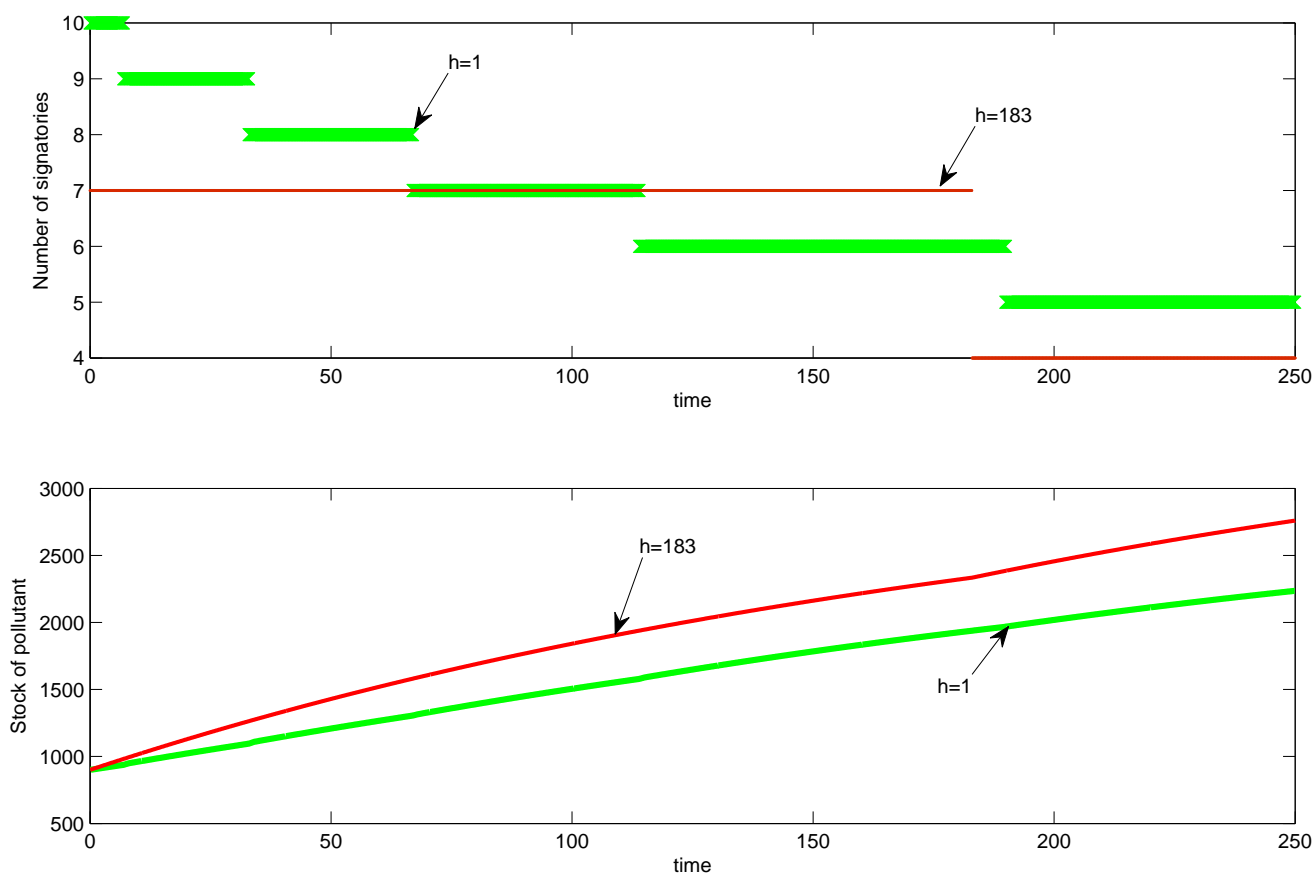

Figure 1: Length of commitment, coalition size, and stock of pollution over time.

The second case is for lengths of commitment $h \in[182.6,194.8]$ used in our simulation. On this interval, simulations suggest two main results. The stock of pollution rises during the first three periods of the game, and afterward decreases and converges asymptotically to its steady state. The coalition size decreases over time and reaches its steady state after a finite number of periods. It always begins with 7 signatories at the initial period and then falls, remaining at 4 signatories for any subsequent period. The type of dynamics obtained in this case is analogous to that for the cooperative equilibrium because the stock of pollution approaches its steady state non monotonically. This is illustrated in Figure 1

\footnotetext{
${ }^{10}$ For other values of $h<182.6$ or $h>194.8$ used in our simulation, we get similar results.
} 
over time interval $[0,250]$ for the case where the length of the period of commitment is equal to $h=18311$ The stock rises from $z_{0}=900$ to $z_{2}=3.2307 \times 10^{3}$ after 3 periods of commitment. After that, since membership remains constant at 4 countries, (23) $-(24)$, and (26) $)$ suggest that it decreases following the dynamic relation $z_{k+1}=0.4005 z_{k}+1918.4$ and converges to its steady state $\bar{z}=3200$ asymptotically.

In the case where $h$ is set to infinity at the initial date, we obtain a coalition of seven signatories as the outcome of the membership game.

Another striking outcome of the simulations is that the trajectory of the pollution stock for $h=1$ always lies above the one that results from any $h<1$ adopted in our simulation 12 Hence, over a given interval of time, any number of negotiation greater than that associated to $h=1$ generates less environmental damages. At every instant, the stock of pollution resulting from the equilibrium with $h=\infty$ is greater than the one we could get from the adoption of some finite lengths of commitment.

Since membership is always decreasing over time, we obtain that for a given length of commitment, the largest coalition is generated at the earliest period of the game. We obtain a non-positive relation between the initial coalition size and the length of commitment, as illustrated by the bottom graph in Figure 2. The rationale is that given the initial stock of pollution $z_{0}$, our simulations have shown that $\bar{n}_{0}\left(z_{0}, h\right)$ is decreasing in $h$. But, $n^{*}\left(z_{0}, h\right)$ is the least integer greater than or equal to $\bar{n}_{0}\left(z_{0}, h\right)$, thus its number integer ceiling $n^{*}\left(z_{0}, h\right)$ is non-increasing in $h$. Over the horizon of the study, such a relation does not exist between the coalition size and the length of commitment. As illustrated in Figure 2, over time interval $[0,60]$, the size of the stable coalition associated to $h=1$ is greater than that associated to

\footnotetext{
${ }^{11}$ Notice that the pollution stock is increasing over time interval $[0,250]$ because this interval is included in the first two periods associated to $h=183$; the illustration covers only the interval $[0,250]$ because of a scale effect. It would not be possible to see the decreasing part of the stock of pollution if Figure 1 were drawn over a very large interval of time. Nevertheless, the dynamics of the stock of pollution associated with its decreasing part has been provided.

${ }^{12}$ Notice that the interval $h<1$ represents values of the length of commitment used in our simulation that are lower than one. Those values are: $h_{p}=(1+p) / 10, p=0,1,2, \ldots, 8$. Moreover, as $p$ rises, the number of times where an IEA is negotiated over a given interval of time $[\delta, \delta+T]$ is non-increasing. Hence, $h<1$ are values of the length of commitment for which the number of negotiation over a given interval is equal or greater than that associated to $h=1$.
} 
$h=183$. However, this result is reverted over the time interval $[130,170]$.
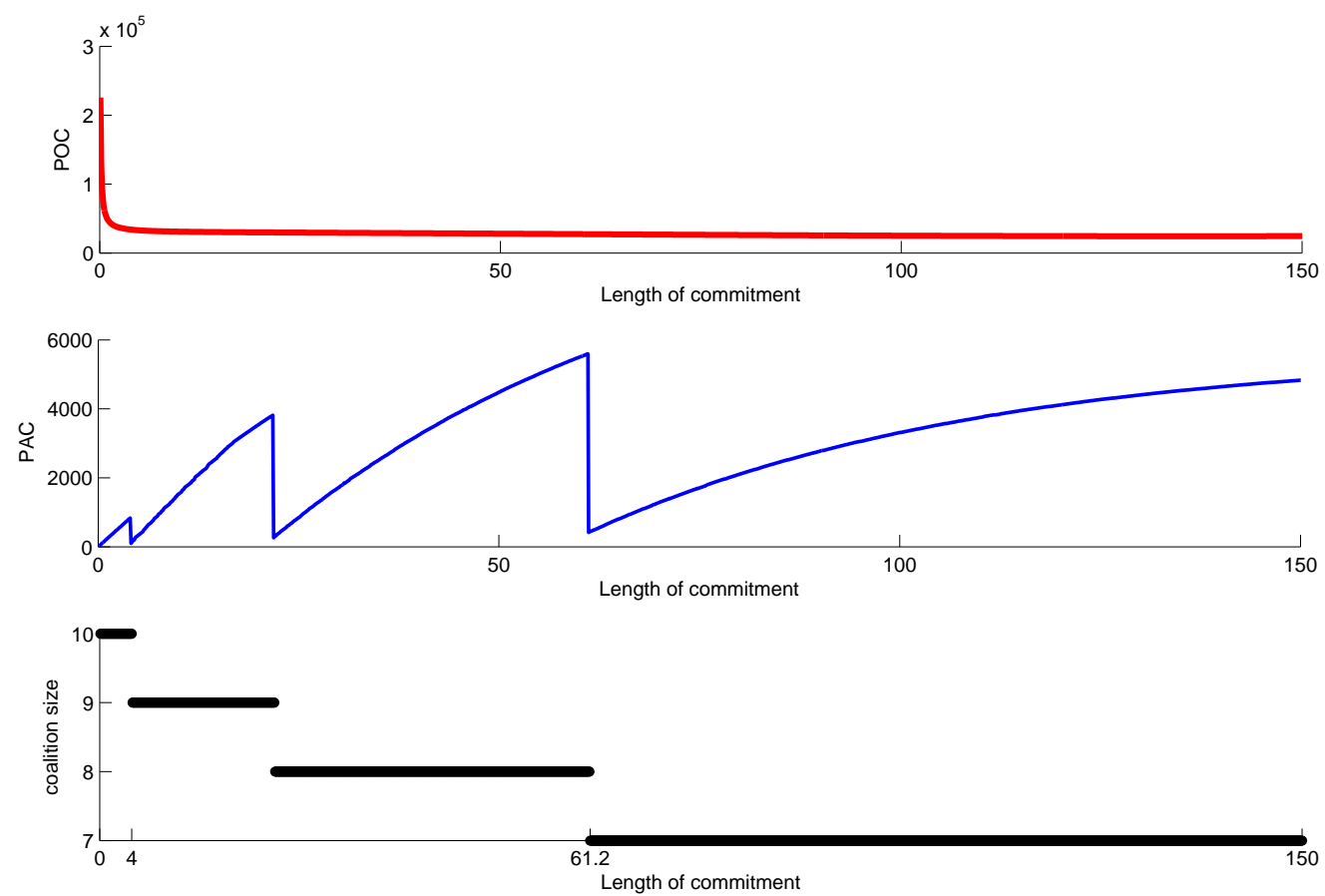

Figure 2: $P O C, P A C$, and coalition size as functions of $h$ at date $t=0$.

\subsection{The length of commitment and the gains from cooperation}

We now consider the effect of the length of commitment on the gain from cooperation.13 It is useful to distinguish the different concepts of gain. The potential gain from cooperation $(P O C)$ is defined as the difference between the sum of the discounted net benefits from cooperative and non-cooperative equilibrium. It is given by:

$$
P O C=V_{i}\left(N, z_{0}, h\right)-V_{j}\left(0, z_{0}, h\right)
$$

The other concept of gain compares the partial cooperative equilibrium to the noncooperative equilibrium. Assume a coalition of $n^{*}\left(z_{0}, h\right)$ signatories. As seen in Section 3 , this results in a decrease in the emission level of signatories and in the aggregate emissions

\footnotetext{
${ }^{13}$ The simulations are done at $t=0$ over $h$ for the initial period only. However, since the rates of emission at each period depend only on the stock of pollution and not explicitly on calendar time, the qualitative results are the same for each subsequent period.
} 
by all countries, as compared to the non-cooperative equilibrium. However, both signatories as well as non-signatories gain from the reduction of global emissions. The partial gain from cooperation by signatories $\left(P A C_{s}\right)$, defined as the difference between the sum of the discounted net benefits by signatories in a stable IEA and in the non-cooperative equilibrium, is given by:

$$
P A C_{s}=V_{i}\left(n^{*}\left(z_{0}, h\right), z_{0}, h\right)-V_{j}\left(0, z_{0}, h\right)
$$

The partial gain from cooperation by non-signatories $\left(P A C_{n s}\right)$ is defined as the difference between the sum of the discounted net benefits by non-signatories given a stable IEA, and that in the non-cooperative equilibrium. It is given by:

$$
P A C_{n s}=V_{j}\left(n^{*}\left(z_{0}, h\right), z_{0}, h\right)-V_{j}\left(0, z_{0}, h\right)
$$

The average partial gain from cooperation by all countries $(P A C)$ is defined as the mean of the $P A C_{s}$ and the $P A C_{n s}$ with the respective weights $n^{*}\left(z_{0}, h\right) / N$ and $1-n^{*}\left(z_{0}, h\right) / N$. Formally, it is given by:

$$
P A C=\frac{n^{*}\left(z_{0}, h\right)}{N} P A C_{s}+\left(1-\frac{n^{*}\left(z_{0}, h\right)}{N}\right) P A C_{n s}
$$

By the definition of external stability, if $n^{*}\left(z_{0}, h\right)=0$, no country can be made better off by cooperating. Hence, in that case, we set $P A C_{s}=P A C_{n s}=P A C=0$.

In Figure 2, the top graph illustrates that $P O C$ is a decreasing function of the length of commitment and that it has a limit, which is the outcome with $h=\infty$.

The bottom graph shows the variations of the stable-coalition size for the initial period with respect to the length of commitment. For $h \in(0,4]$, the potential gain from cooperation is sufficiently large, leading to a maximum level of cooperation of 10 countries. For $h \in$ $(4,61.2)$, the potential gain from cooperation declines from a not very large to a not very small value generating a non-positive relation between the stable coalition size of the initial period and the length of commitment. For $h \geq 61.2$, the potential gain from cooperation takes only small values, generating a stable coalition of seven members for the initial period. 
In particular, taking the limit of $n^{*}\left(z_{0}, h\right)$ as $h$ goes to infinity, we obtain a seven-signatories coalition as the outcome of the $h=\infty$ coalition size. The intuition underlying these results is that at the initial date, shrinking the length of commitment raises the potential gain from cooperation (i.e., the maximum payoff that a country could derive from adhering to a coalition), which increases the incentive to ratify the treaty. It is interesting to note that while in the static model of Barrett (1994), an IEA may result in a significant level of cooperation only if the $P O C$ is very small; in our model this pessimistic result need not hold for some values of the length of commitment. Indeed, as shown in Figure 2, the length of commitment that maximizes the POC also sustains the highest level of cooperation.
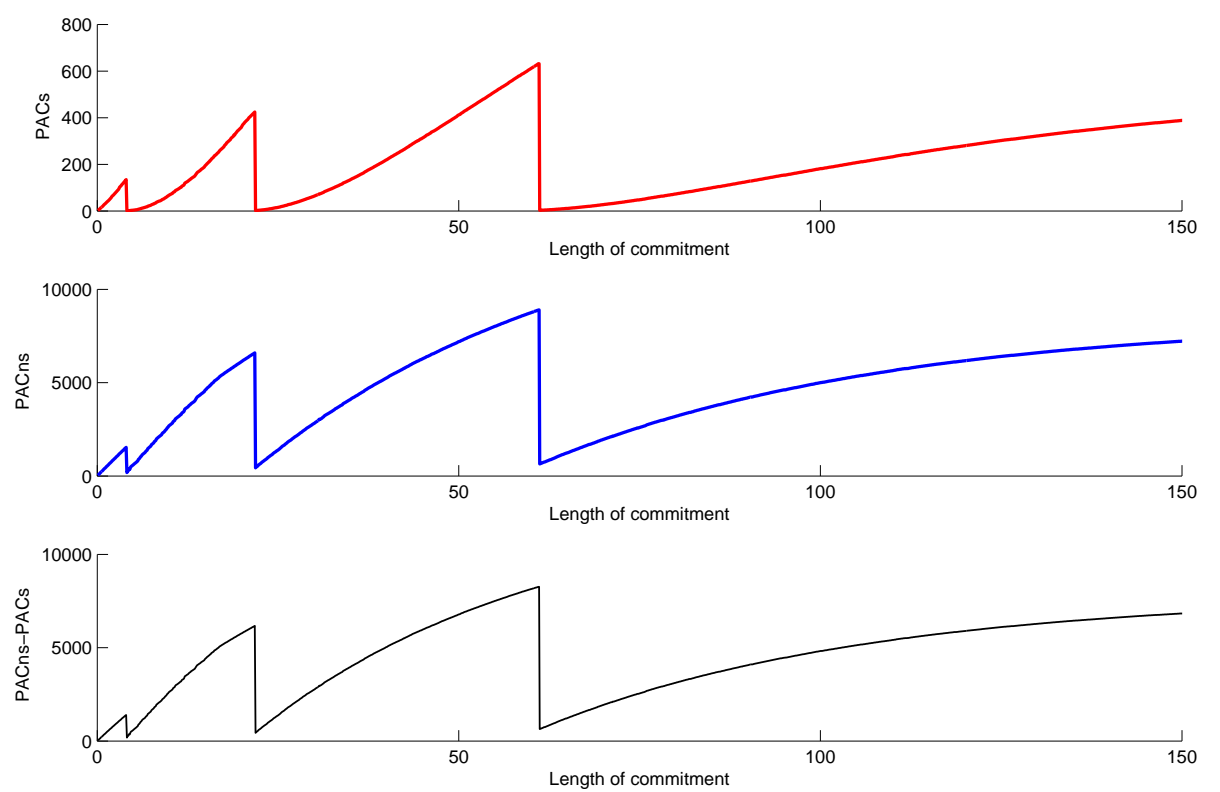

Figure 3: Gain from cooperation by signatories and by non-signatories as functions of $h$.

The $P A C_{s}, P A C_{n s}$, and the $P A C$ are all piecewise increasing functions of $h$ as illustrated in Figure 2 and Figure 3. They depend on the coalition size, and each of them has as limit the outcome with $h=\infty$. Because non-signatories gain more than signatories from any cooperation, it follows that $P A C_{s} \geq P A C_{n s}$ for all values of the length of commitment, as shown in the bottom graph of Figure 3, These results indicate that as the length of 
commitment is increased, the number of signatories tends to decrease, whereas each of $P A C_{s}$, $P A C_{n s}$, and $P A C$ follows a non-monotonic evolution.

In spite of the fact that we cannot claim any general result, the above discussion suggests that for some lengths of commitment $h \neq 1$ the gain from cooperation is higher than for $h=1$, and that some finite lengths of commitment can, for each of $P O C, P A C, P A C_{s}$, and $P A C_{n s}$, sustain a higher value than by letting the length of commitment to go to infinity. It is clear that the length of commitment significantly affects the size of stable coalitions and the gains from cooperation.

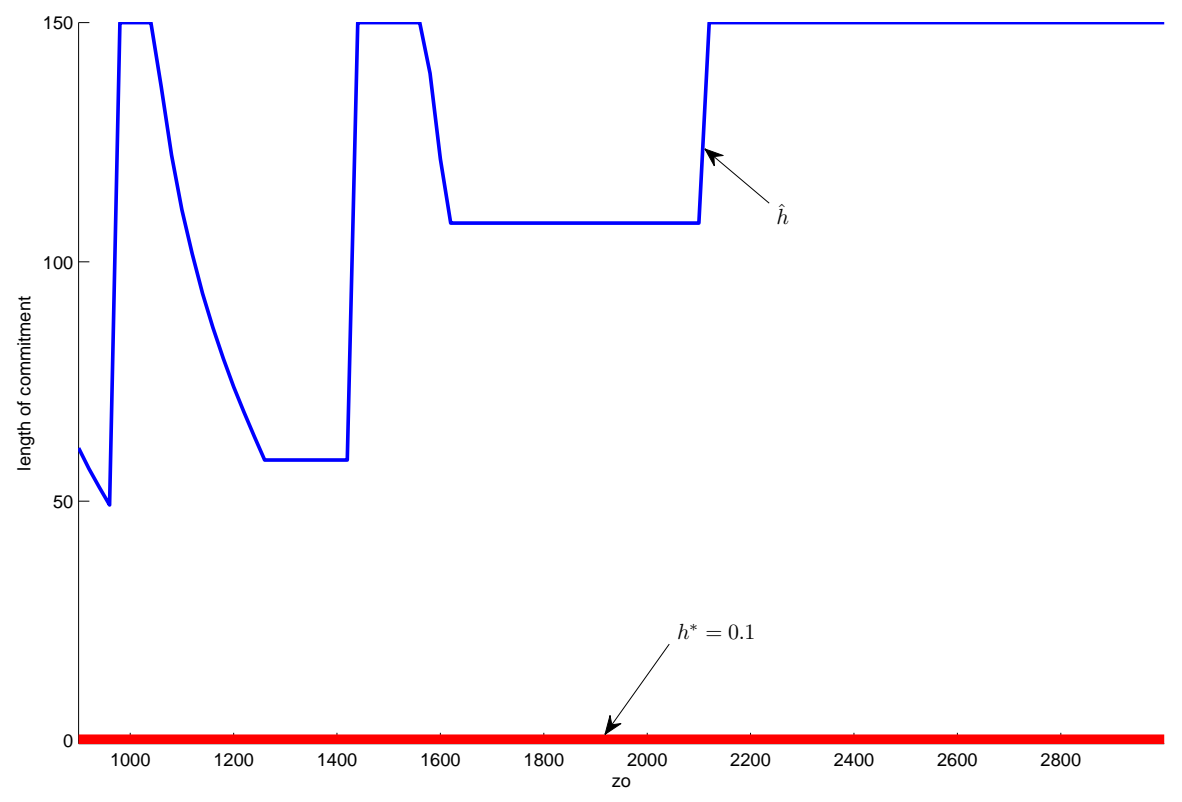

Figure 4: $\hat{h}=\arg \max _{h} P A C_{s}$ versus $h^{*}=\arg \min _{h} Q$.

Finally, it is useful to examine the relation between the initial stock of any given period of commitment $\left(z_{k}\right)$, the length of commitment that maximizes the $P A C_{s}$ (denote it $\hat{h}$ ), and the length of commitment that can sustain the minimum aggregate emissions (denote it $h^{*}$ ). To do this, we have first simulated 8001 values of the current pollution stock following the replication $z_{k}=z_{k-1}+20, k=1, \ldots, 105 ; z_{0}=900$. For each of these values, we calculate $h^{*}$ and $\hat{h}$. The top curve of Figure 4 illustrates that $\hat{h}$ is a piecewise continuous function of the 
initial stock of the period of commitment. This is because its argument $n^{*}\left(z_{0}, h\right)$ is a piecewise function. The bottom curve shows that $h^{*}$ is independent of the initial pollution stock of the period of commitment. Furthermore, $\hat{h}$ is always greater than $h^{*}$. These results highlight the difficulties of reconciling the private gain from cooperation and the best protection of the environment.

\section{Conclusion}

The existing literature on dynamic International Environmental Agreements has relied on one of two approaches. The first consists in assuming that membership and emission strategies are determined once and for all, as a function of time, at the outset of an infinite horizon. The other consists in analyzing the problem in a discrete-time framework and assuming that membership and emission decisions are revised at the beginning of each period, whose length has been arbitrarily set equal to one. This paper has explored the middle ground by treating the length of the period of commitment as a positive parameter and studying the effect of varying this parameter on the size of stable International Environmental Agreements. It has been shown that the length of the period of commitment can have considerable impacts on the size of stable International Environmental Agreements. The results suggest at the initial date that for very large lengths of commitment, only small stable coalitions can be sustained. But, below some threshold, as the length of the period of commitment is decreased, the size of the stable coalition tends to increase. It does so until, if this length is sufficiently small, the largest level of cooperation can be attained.

Since our results rest on particular functional forms and on numerical simulations, there is no claim to generality. However, they do clearly show that the length of the period of commitment can have very significant effects on the outcome of International Environmental Agreements. This suggests that considerable attention should be devoted to the determination of the length of the period of commitment in discussions of this type of international treaties. 
For the purpose of this paper, it has been sufficient to treat the length of commitment as a parameter. However, how best to determine the length of commitment is another matter, which is left for further research. 


\section{Appendix}

\section{A proof of Condition 7}

Since, by assumption, the $q_{i}, i=1, \ldots, N$ are time stationary over interval $[k h,(k+1) h)$, so is $Q=\sum_{i=1}^{N} q_{i}$. Using this result along with (2) and (5), we get

$$
\int_{k h}^{(k+1) h} e^{-r(s-k h)} \pi(q, z(s)) d s=a q f(r, h)+D\left(Q, z_{k}\right),
$$

where $D\left(Q, z_{k}\right)=-\frac{\gamma}{2}\left[\left(\frac{Q}{\rho}\right)^{2} f(r, h)+\left(z_{k}-\frac{Q}{\rho}\right)^{2} f(r+2 \rho, h)+2 \frac{Q}{\rho}\left(z_{k}-\frac{Q}{\rho}\right) f(r+\rho, h)\right]$ and where $f(x, h)=\left(1-e^{-h x}\right) / x$ for all $x>0$.

\section{B The fully-cooperative equilibrium}

The value function $V_{N}(z)=N V_{i}(z)$ satisfies the Bellman equation:

$$
N V_{i}(z)=\max _{q_{i}, i \in N}\left\{\sum_{i=1}^{N} a q_{i} f(r, h)+N D(Q, z)+N e^{-r h} V_{i}\left(Q f(\rho, h)+z e^{-\rho h}\right)\right\},
$$

where $Q=\sum_{i}^{N} q_{i}$. The quadratic nature of the benefit function in $z$ suggests the guess: $V_{i}(z)=-\bar{A} z^{2} / 2 N-\bar{B} z / N+\bar{C} / N$. Using (11)-(13) for $\Psi=V_{i}, n=N$ and for $q_{i} \in(0,1)$ we get:

$$
q_{i}\left(z_{k}\right)=\frac{a f(r, h)-\bar{B} N f(\rho, h) e^{-r h}-N z_{k}\left(\lambda_{2}+\bar{A} f(\rho, h) e^{-h(r+\rho)}\right)}{N^{2}\left[\lambda_{1}+\bar{A} f(\rho, h)^{2} e^{-r h}\right]} .
$$

Substituting (28) into the Bellman equation and using the envelope theorem, we obtain:

$$
V_{i}^{\prime}(z)=-z \gamma f(r+2 \rho, h)-N \lambda_{2} q_{i}(z)+e^{-h(r+\rho)} V_{i}^{\prime}\left(N f(\rho, h) q_{i}(z)+z e^{-\rho h}\right), \forall z \geq 0 .
$$

Equating the coefficients of the $L H S$ and $R H S$ of this first-degree polynomial in $z$, we find that

$$
\bar{A}=\left[-\bar{a}_{1}+\sqrt{\bar{a}_{1}^{2}-4 \bar{a}_{2} \bar{a}_{0}}\right] / 2 \bar{a}_{2},
$$

which is the positive root of the second degree polynomial $\bar{a}_{2} A^{2}+\bar{a}_{1} A+\bar{a}_{0}=0$, where,

$$
\begin{aligned}
& \bar{a}_{2}=f(\rho, h)^{2} e^{-r h}, \\
& \bar{a}_{1}=\lambda_{1}\left(1-e^{-h(r+2 \rho)}\right)+\lambda_{2}(1+N) f(\rho, h) e^{-h(r+\rho)}-N \gamma f(r+2 \rho, h) f(\rho, h)^{2} e^{-r h}, \\
& \bar{a}_{0}=N\left[\lambda_{2}^{2}-\gamma \lambda_{1} f(r+2 \rho, h)\right] .
\end{aligned}
$$


Given $\bar{A}$, we find:

$$
\bar{B}=\frac{a f(r, h)\left(N \lambda_{2}+\bar{A} f(\rho, h) e^{-h(r+\rho)}\right)}{N\left(\lambda_{1}+\bar{A} f(\rho, h)^{2} e^{-r h}\right)\left(1-e^{-h(r+\rho)}\right)+N f(\rho, h) e^{-r h}\left(N \lambda_{2}+\bar{A} f(\rho, h) e^{-h(r+\rho)}\right)} .
$$

Given $\bar{A}$ and $\bar{B}$, using (27) at the optimum, we get

$\bar{C}=N \bar{\beta}\left[a f(r, h)-\bar{B} f(\rho, h) e^{-r h}\right] /\left(1-e^{-r h}\right)-N^{2} \bar{\beta}^{2}\left[N \lambda_{1}+\bar{A} f(\rho, h)^{2} e^{-r h}\right] / 2\left(1-e^{-r h}\right)$,

where $\bar{\beta}=\left[a f(r, h)-N \bar{B} f(\rho, h) e^{-r h}\right] / N^{2}\left[\lambda_{1}+\bar{A} f(\rho, h)^{2} e^{-r h}\right]$.

The dynamic evolution of the pollution stock is then

$$
\begin{aligned}
z_{k+1} & =f(\rho, h) \frac{a f(r, h)-\bar{B} N f(\rho, h) e^{-r h}-z_{k} N\left(\lambda_{2}+\bar{A} f(\rho, h) e^{-h(r+\rho)}\right)}{N \lambda_{1}+N \bar{A} f(\rho, h)^{2} e^{-r h}}+z_{k} e^{-\rho h} \\
& \equiv \varphi_{N}\left(z_{k}\right) .
\end{aligned}
$$

The unique solution to the equation $x=\varphi_{N}(x)$ is:

$$
\bar{z}=\frac{a f(r, h)-\bar{B} N e^{-r h} f(\rho, h)}{N\left(\lambda_{2}+\bar{A} f(\rho, h) e^{-r h}\right)+\rho N\left[\lambda_{1}+\bar{A} f(\rho, h)^{2} e^{-r h}\right]}
$$

Hence, $\forall k=0,1,2,3, \ldots$,

$$
z_{k+1}-\bar{z} \equiv \varphi_{N}\left(z_{k}\right)-\bar{z}=R_{N}\left(z_{k}-\bar{z}\right)
$$

so that

$$
z_{k}=\bar{z}+\left(R_{N}\right)^{k}\left(z_{0}-\bar{z}\right), \quad \forall \quad k=0,1,2,3, \ldots,
$$

where,

$$
R_{N}=\frac{\lambda_{1} e^{-\rho h}-\lambda_{2} f(\rho, h)}{\lambda_{1}+\bar{A} f(\rho, h)^{2} e^{-r h}}
$$

We have $1>R_{N}$. Indeed, because all the parameters are non-negative and $\bar{A}>0$, we have the following inequality:

$$
\left(1-e^{-\rho h}\right) \lambda_{1}+\bar{A} f(\rho, h)^{2} e^{-r h}+\lambda_{2} f(\rho, h)>0 .
$$

Rearranging the terms of this inequality, we get:

$$
\lambda_{1}+\bar{A} f(\rho, h)^{2} e^{-r h}>\lambda_{1} e^{-\rho h}-\lambda_{2} f(\rho, h) .
$$


Dividing the LHS and the RHS of the last inequality by its LHS, we obtain $1>R_{N}$. The sequence $z_{k}-\bar{z}$ defined in (29) being a geometric progression, a necessary and sufficient condition for $z_{k}$ to converge is $1>R_{N}>-1$. It has been established that $1>R_{N}$. Therefore, if and only if $R_{N}>-1, z_{k}$ converges and its limit is $\bar{z}$. It converges monotonically if $R_{N}>0$. The steady-state emission rate exists if and only $R_{N}>-1$ and is given by:

$$
\bar{q}_{i}=\frac{a f(r, h)-N \bar{B} f(\rho, h) e^{-r h}-\bar{z} N\left[\lambda_{2}+\bar{A} f(\rho, h) e^{-h(r+\rho)}\right]}{N^{2}\left[\lambda_{1}+\bar{A} f(\rho, h)^{2} e^{-r h}\right]} .
$$

Notice that we have $0<q_{i}\left(z_{k}\right)<1$. Indeed, let us assume that inequalities from (17) hold. On the one hand, we must have

$a f(r, h)<N^{2}\left[\lambda_{1}+\bar{A} e^{-r h} f(\rho, h)^{2}\right]+N \bar{B} f(\rho, h) e^{-r h}+z_{k} N\left[\lambda_{2}+\bar{A} f(\rho, h) e^{-h(r+\rho)}\right]$ which, rearranging, yields $a f(r, h)-N \bar{B} f(\rho, h) e^{-r h}-z_{k} N\left[\lambda_{2}+\bar{A} f(\rho, h) e^{-h(r+\rho)}\right]<N^{2}\left[\lambda_{1}+\bar{A} e^{-r h} f(\rho, h)^{2}\right]$. Dividing the two sides of this inequality by its RHS, one gets $q_{i}\left(z_{k}\right)<1$. On the other hand, it is easy to show that

$a f(r, h)-N \bar{B} f(\rho, h) e^{-r h} \geq N\left[\lambda_{2}+\bar{A} f(\rho, h) e^{-h(r+\rho)}\right] \bar{z}$. Since from (29) we have $\bar{z}>z_{2 k} \geq 0$, it then follows that af $(r, h)-N \bar{B} f(\rho, h) e^{-r h}>N\left[\lambda_{2}+\bar{A} f(\rho, h) e^{-h(r+\rho)}\right] z_{2 k}$. Rearranging this inequality, we can see that the numerator of (28) is positive at the point $z_{2 k}$. Since the denominator of (28) is always positive, we can conclude that $q_{i}\left(z_{2 k}\right)>0$. Unfortunately, we have not been able to prove analytically that $q_{i}\left(z_{2 k+1}\right)>0, k=0,1,2, \ldots$ However, we take into account this constraint in our simulations.

\section{Proof of statements in Section 4}

Making use of (10) and the quadratic approximation $\Psi(z)=-\frac{A}{2} z^{2}-B z+C$, for all $z$, non-signatories will emit one at each period if and only if

$$
a f(r, h) \geq \lambda_{1} Q+z_{k} \lambda_{2}+f(\rho, h) e^{-r h}\left\{B+A\left[f(\rho, h) Q+z_{k} e^{-\rho h}\right]\right\}
$$

Notice that, $Q \leq N ; z_{k} \leq N / \rho$ and $f(\rho, h) Q+z_{k} e^{-\rho h} \leq N / \rho$. Replacing $Q, z_{k}$ and $f(\rho, h) Q+z_{k} e^{-\rho h}$ by their upper bound in (30) yields

$$
a f(r, h) \geq N\left(\lambda_{1}+\lambda_{2} / \rho\right)+f(\rho, h) e^{-r h}(B+A N / \rho)
$$


which is condition (19). Thus if (19) holds, it will be optimal for a non-signatory to play $q_{j}=1$ at each period.

Given the quadratic approximation of $\Psi$, for $q_{j}=1$, relations (11)-(13) can be rewritten as

$$
\begin{aligned}
& a f(r, h) \leq-n^{2}\left[\lambda_{1}+A e^{-r h} f(\rho, h)^{2}\right]+n \tau\left(z_{k}, h\right), q_{i}=0 \\
& a f(r, h)=n^{2} q_{i}\left[\lambda_{1}+A e^{-r h} f(\rho, h)^{2}\right]-n^{2}\left[\lambda_{1}+A e^{-r h} f(\rho, h)^{2}\right]+n \tau\left(z_{k}, h\right), 0 \leq q_{i} \leq 1 \\
& a f(r, h) \geq n \tau\left(z_{k}, h\right), q_{i}=1 .
\end{aligned}
$$

Solving (34), we get: $q_{i}\left(n, z_{k}\right)=1$ if and only if $1 \leq n \leq n_{0}\left(z_{k}\right) \equiv a f(r, h) / \tau\left(z_{k}, h\right)$.

Pick $\bar{n}_{1}\left(z_{k}\right)$ and $\bar{n}_{2}\left(z_{k}\right)$ to be the two roots of the second degree equation in $n:-n^{2}\left[\lambda_{1}+\right.$ $\left.A e^{-r h} f(\rho, h)^{2}\right]+n \tau\left(z_{k}, h\right)=a f(r, h)$. Their expressions are given by (21). Solving (32) yields $q_{i}\left(n, z_{k}\right)=0$ if and and only if we have $\bar{n}_{1}\left(z_{k}\right) \leq n \leq \bar{n}_{2}\left(z_{k}\right)$.

Solving (33), we obtain

$$
0 \leq q_{i}\left(n, z_{k}\right)=\frac{a f(r, h)+n^{2}\left(\lambda_{1}+A e^{-r h} f(\rho, h)^{2}\right)-n \tau\left(z_{k}, h\right)}{n^{2}\left(\lambda_{1}+A e^{-r h} f(\rho, h)^{2}\right)} \leq 1,
$$

if and only if $n \in\left[\bar{n}_{0}\left(z_{k}\right), \bar{n}_{1}\left(z_{k}\right)\right]$.

As illustrated in Figure $5, \bar{n}_{1}\left(z_{k}\right)$ and $\bar{n}_{2}\left(z_{k}\right)$ are two positive real numbers but only if there exists $n \in(0, N)$ for which: $-n^{2}\left[\lambda_{1}+A e^{-r h} f(\rho, h)^{2}\right]+n \tau\left(z_{k}, h\right)$ lies above $a f(r, h)$. In addition, $\bar{n}_{1}\left(z_{k}\right)<N<\bar{n}_{2}\left(z_{k}\right)$ is satisfied only when we have the following:

$$
-N^{2}\left[\lambda_{1}+A e^{-r h} f(\rho, h)^{2}\right]+N \tau\left(z_{k}, h\right)>a f(r, h) .
$$

Notice that $(i) \tau(z, h)$ is increasing in $z ;(i i)$ our simulations show that for $z_{0}$ lying below the steady state of the non-cooperative equilibrium: $N / \rho$, the stock of pollution increases over time so that its lower bound is $z_{0} ;($ iii $) z_{0}<N / \rho$, implies that $\tau\left(z_{k}, h\right) \geq \tau\left(z_{0}, h\right)$, $k=0,1,2 \ldots ;(i v)$ if we replace $z_{k}$ by $z_{0}$ in (36), we will get exactly the condition in (20). Thus if the condition $-N^{2}\left[\lambda_{1}+A e^{-r h} f(\rho, h)^{2}\right]+N \tau\left(z_{0}, h\right)>a f(r, h)$ holds, it will be so for (36). The inequality (20) is then a sufficient condition to have $\bar{n}_{1}\left(z_{k}\right)<N<\bar{n}_{2}\left(z_{k}\right)$, $k=0,1,2 \ldots$ 
Since we have: $n \tau\left(z_{k}, h\right) \geq-n^{2}\left[\lambda_{1}+A e^{-r h} f(\rho, h)^{2}\right]+n \tau\left(z_{k}, h\right)$, when conditions (19)-(20) hold, we also have $\bar{n}_{0}\left(z_{k}\right) \leq \bar{n}_{1}\left(z_{k}\right)$, as illustrated in Figure 5. Finally, making use of $Q \leq N$; $z_{k} \leq \tilde{z}$ for all $k$, we have shown that $\bar{n}_{0}\left(z_{k}\right) \geq 1$ when the condition (20) is satisfied.

Summarizing, if conditions (19)-(20) are satisfied, we must have two results. On the one hand, we must have: $1 \leq \bar{n}_{0}\left(z_{k}\right)<\bar{n}_{1}\left(z_{k}\right)<N<\bar{n}_{2}\left(z_{k}\right)$. On the other hand, non-signatories must emit one at each period, while the typical signatory's decision rule for emissions will be given by (23) .

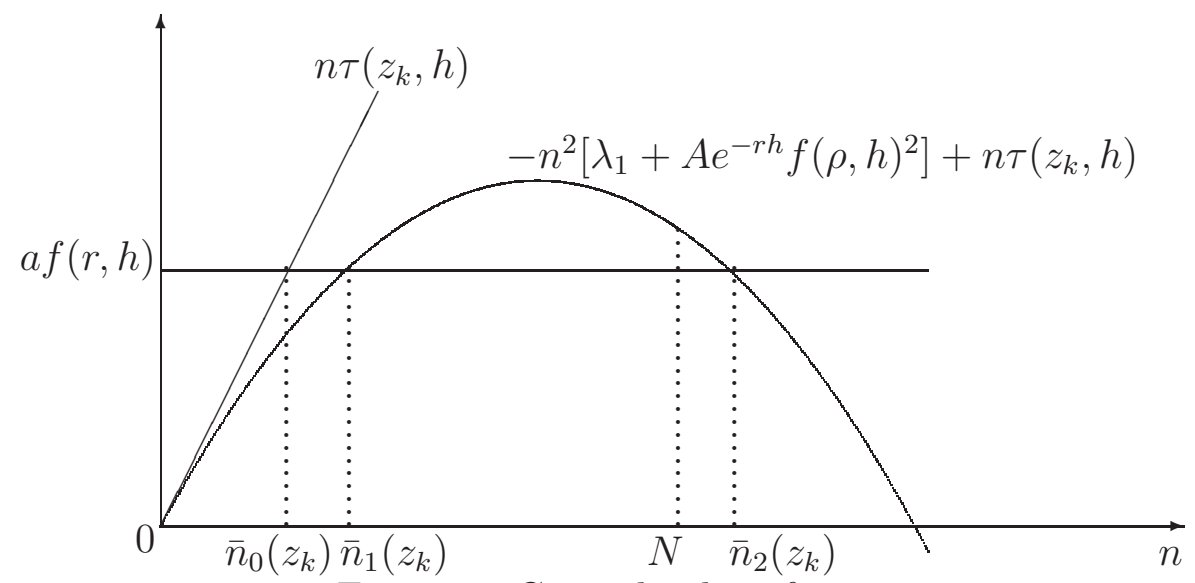

Figure 5: Critical values for $n$.

\section{The algorithm}

This section presents the algorithm used to approximate the value function $\Psi$. It is made up of the eight following steps.

(i) Generate values of $h$, using the formula, $h_{p}=h_{p-1}+0.1 ; p=1, \ldots, 2499$, with $h_{0}=0.1$. We choose plausible parameters $a, \gamma, r, \rho, z_{0}$, and $N$ such that the conditions (16), (17), (19), and (201) always hold for all $h_{p}, p=0, \ldots, 2499$.

(ii) For each vector $\left(a, \gamma, r, \rho, z_{0}, N, h_{p}\right)$ of parameters we consider as initial values for $A, B$ and $C$ the values of the quadratic value function for the non-cooperative equilibrium.

(iii) We generate 251 values of the stocks $z$ in the interval $\left[z_{0}, \tilde{z}\right]$ following the sequence: $z_{p}=z_{0}+p\left(\tilde{z}-z_{0}\right) / 250 ; p=0, \ldots, 250$, where $\tilde{z}$ represents the steady state of the pollution stock for the non-cooperative equilibrium. 
(iv) For each value of $z$ from $(i i i)$, compute $\bar{n}_{1}(z)$ and $\bar{n}_{2}(z)$ using (21), $\bar{n}_{0}(z)$ using (22), and $q_{i}(n, z)$ using (23). Using (14) and (15), compute $\Lambda(n, z)=V_{i}(n, z)-V_{j}(n-1, z)$, for $\Psi(z)=-A z^{2} / 2-B z+C$.

$(v)$ For each value of $z$ we calculate $n^{*}(z)$, the highest value of $n$ for which $\Lambda(n, z) \geq 0$.

(vi) We then compute for each of the 251 values of $z, n^{*}(z)$ and $q_{i}\left(n^{*}(z), z\right)$. We estimate the relation $Q\left(n^{*}(z), z\right) \approx \beta+\alpha z$ by linear regression that yields an estimation of $(\beta, \alpha)$.

(vii) Replacing $Q\left(n^{*}(z), z\right)$ by $\beta+\alpha z$ in (18) and equating the coefficient of power of $z$, we get the new estimation of $A, B$, and $C$, which are the following:

$$
\begin{aligned}
& A=\left[\alpha^{2} \lambda_{1}+2 \alpha \lambda_{2}+\gamma f(r+2 \rho, h)\right] /\left[1-e^{-r h}\left(e^{-\rho h}+\alpha f(\rho, h)\right)^{2}\right], \\
& B=\frac{N \beta\left(\alpha \lambda_{1}+\lambda_{2}\right)-a \alpha f(r, h)+N A \beta e^{-r h} f(\rho, h)\left(e^{-\rho h}+\alpha f(\rho, h)\right)}{N\left[1-e^{-r h}\left(e^{-\rho h}+\alpha f(\rho, h)\right)\right]}, \\
& C=\frac{2 a \beta f(r, h)-N \beta^{2} \lambda_{1}-N \beta e^{-r h} f(\rho, h)(2 B+A \beta f(\rho, h))}{2 N\left(1-e^{-r h}\right)} .
\end{aligned}
$$

(viii) We repeat this operation until convergence is obtained, i.e., variations of $A, B$, and $C$ not greater than $5 \%$ or the number of iteration not greater than 101 . 


\section{References}

Barrett, S. (1994) 'Self-enforcing International Environmental Agreements.' Oxford Econ. Pap. 46, 878-894

Carraro, C., and D. Siniscalco (1993) 'Strategies for the international protection of the environment.' J. Public Econ 52, 309-328

D'Aspremont, C., A. Jacquemin, J.J. Gabszewicz, and J. Weymark (1983) 'On the stability of collusive price leadership.' Canadian Journal of Economics 16, 17-25

de Zeeuw, Aart (2008) 'Dynamic effects on the stability of international environmental agreements.' Journal of Environmental Economics and Management 55, 163-174

Dockner, Engelbert J., and Ngo Van Long (1993) 'International pollution control: Cooperative versus Noncooperative Strategies.' Journal of Environmental Economics And Management 24, 13-29

Long, Ngo Van (1992) 'Pollution control: A differential game approach.' Annals of Operations Research 37, 283-296

Nkuiya, Bruno (2011) 'International emission strategies under the threat of a sudden jump in damages.' Cahiers de recherche CREATE 2011-1, Département d'économique, Université Laval

Nkuiya, Bruno, Walid Marrouch, and Eric Bahel (2011) 'International environmental agreements under endogenous uncertainty.' Working papers e07-32, Virginia Polytechnic Institute and State University, Department of Economics

Pavlova, Yulia (2010) 'How rapid should emission reduction be? A game-theoretic approach.' Natural Resource Modeling 23, 540-564 
Reinganum, Jennifer F., and Nancy L. Stokey (1985) 'Oligopoly extraction of a common property natural resource: The importance of the period of commitment in dynamic games.' International Economic Review 26, 161-173

Rubio, Santiago J., and A. Ulph (2007) 'An infinite-horizon model of dynamic membership of international environmental agreements.' Journal of Environmental Economics and Management 54, 296-310

Rubio, Santiago J., and Begoña Casino (2005) 'Self-enforcing international environmental agreements with a stock of pollutant.' Spanish Economic Review 7, 89-109

Ulph, Alistair (2004) 'Stable International Environmental Agreements with a Stock Pollutant, Uncertainty and Learning.' The Journal of Risk and Uncertainty 29, 53-73 